\title{
Infinitely many solutions for $p$-harmonic equation with singular term
}

Huazhao Xie ${ }^{1 *}$ and Jianping Wang ${ }^{2}$

\section{${ }^{\text {*Correspondence: }}$}

hzh_xie@yahoo.com.cn

'Department of Mathematics, Henan University of Economics and Law, Zhengzhou, 450002, China Full list of author information is available at the end of the article

\section{Abstract}

In this paper, we study the following $p$-harmonic problem involving the Hardy term:

$$
\Delta\left(|\Delta u|^{p-2} \Delta u\right)-\lambda \frac{|u|^{p-2} u}{|x|^{2 p}}=f(x, u), \quad \text { in } \Omega, \quad u=\frac{\partial u}{\partial n}=0 \quad \text { on } \partial \Omega
$$

where $\Omega$ is an open bounded domain containing the origin in $\mathbb{R}^{N}, 1<p<\frac{N}{2}$ and $0 \leq \lambda<\left[N(p-1)(N-2 p) / p^{2}\right]^{p}$. By using the variational method, we prove that the above problem has infinitely many solutions with positive energy levels.

AMS Subject Classification: 35J60; 35J65

Keywords: infinitely many solutions; $p$-harmonic; Hardy term

\section{Introduction}

The main purpose of this paper is to show the existence of infinitely many solutions for the following $p$-harmonic equation:

$$
\begin{cases}\Delta\left(|\Delta u|^{p-2} \Delta u\right)-\lambda \frac{|u|^{p-2} u}{|x|^{2 p}}=f(x, u), & x \in \Omega, \\ u=\frac{\partial u}{\partial n}=0, & x \in \partial \Omega,\end{cases}
$$

where $\Omega$ is an open bounded domain containing the origin in $\mathbb{R}^{N}$, the boundary $\partial \Omega$ is smooth. $1<p<\frac{N}{2}, 0 \leq \lambda<\bar{\lambda}=\left[N(p-1)(N-2 p) / p^{2}\right]^{p}$. $\frac{\partial}{\partial n}$ is the outer normal derivative. Nonlinearity $f(x, u)$ satisfies the following conditions:

$\left(\mathrm{f}_{1}\right) f(x, u)$ is continuous on $\Omega \times \mathbb{R}$ and limits subcritical growing at infinity; that is,

$$
\lim _{u \rightarrow \infty} \frac{f(x, u)}{|u|^{p^{n}-1}}=0, \quad \text { uniformly for } x \in \Omega
$$

where $p^{\prime \prime}=N p /(N-2 p)$ is the critical exponent of Sobolev's embedding $W_{0}^{2, p}(\Omega) \hookrightarrow L^{s}(\Omega)$.

$\left(\mathrm{f}_{2}\right)$ For any $x \in \Omega, f(x, u)$ satisfies

$$
\lim _{u \rightarrow 0} \frac{f(x, u) u}{|u|^{p}}=0 \text { and } \quad \lim _{u \rightarrow \infty} \frac{f(x, u) u}{|u|^{p}}=+\infty .
$$


$\left(\mathrm{f}_{3}\right)$ Denote $G(x, t)=f(x, t) t-p \int_{0}^{t} f(x, s) d s$. For all $x \in \Omega$, there exists a constant $M \geq 0$ such that

$$
G\left(x, t_{1}\right) \leq G\left(x, t_{2}\right) \quad \text { for any } M \leq\left|t_{1}\right| \leq\left|t_{2}\right| \text {. }
$$

$\left(\mathrm{f}_{4}\right) f(x, u)$ is odd with respect to $u$.

By the Hardy-Rellich inequality (see $[1,2])$, we know that

$$
\int_{\Omega} \frac{|u|^{p}}{|x|^{2 p}} d x \leq \frac{1}{\bar{\lambda}} \int_{\Omega}|\Delta u|^{p} d x
$$

Obviously, for any $\lambda \in[0, \bar{\lambda})$,

$$
\left(1-\frac{\lambda}{\bar{\lambda}}\right) \int_{\Omega}|\Delta u|^{p} d x \leq \int_{\Omega}\left(|\Delta u|^{p}-\lambda \frac{|u|^{p}}{|x|^{2 p}}\right) d x \leq \int_{\Omega}|\Delta u|^{p} d x .
$$

In $W_{0}^{2, p}(\Omega)$, for $\lambda \in[0, \bar{\lambda})$, we define

$$
\|u\|=\|u\|_{W_{0}^{2, p}(\Omega)}:=\left(\int_{\Omega}\left(|\Delta u|^{p}-\lambda \frac{|u|^{p}}{|x|^{2 p}}\right) d x\right)^{\frac{1}{p}}
$$

this norm is equivalent to $\left(\int_{\Omega}|\Delta u|^{p} d x\right)^{1 / p}$.

A weak solution of the problem (1.1) is a critical point of the energy functional

$$
I(u)=\frac{1}{p} \int_{\Omega}\left(|\Delta u|^{p}-\lambda \frac{|u|^{p}}{|x|^{2 p}}\right) d x-\int_{\Omega} F(x, u) d x, \quad u \in W_{0}^{2, p}(\Omega),
$$

where $F(x, u)=\int_{0}^{u} f(x, s) d s$. It is easy to check that $I(u)$ is a continuous even functional.

Biharmonic equations can describe the static form change of a beam or the sport of a rigid body. For example, this type of equation furnishes a model for studying traveling wave in suspension bridges (see [3]). By using variational arguments, many authors investigated nonlinear biharmonic equations under Dirichlet boundary conditions or Navier boundary conditions and got interesting results (see [4-10]).

$\mathrm{Li}$ and Squassina in [11] considered the superlinear $p$-harmonic equation with Navier boundary conditions

$$
\Delta\left(|\Delta u|^{p-2} \Delta u\right)=f(x, u), \quad \text { in } \Omega, \quad u=\Delta u=0 \quad \text { on } \partial \Omega,
$$

where $\Omega$ is an open bounded domain in $\mathbb{R}^{N}$ with a smooth boundary $\partial \Omega . p>1, N \geq 2 p+1$ and $f: \Omega \times \mathbb{R} \rightarrow \mathbb{R}$ is a Carathéodory function such that for some positive constant $C$,

$$
|f(x, u)| \leq C\left(1+|u|^{q-1}\right) \quad \text { for all } q \in\left[1, p^{*}\right)
$$

By means of the Morse theory, they proved the existence of two nontrivial solutions to (1.7). After that, Li and Tang [12] considered a more general problem than (1.7). They got three solutions by the three critical points theorem which was obtained in [13]. If $f(x, u)=\lambda g(x, u)$, Candito and Bisci [14] established a well-determined interval of values of the parameter $\lambda$ for which the problem (1.7) admits at least two distinct weak solutions. 
The authors in [15] considered the $p$-harmonic equation with Dirichlet conditions and obtained the existence of a nontrivial solution. Under the condition of (1.8), [16] proved the existence and multiplicity of weak solutions for the nonuniformly nonlinear problem

$$
\Delta(a(x, \Delta u))=f(x, u), \quad \text { in } \Omega, \quad u=\frac{\partial u}{\partial n}=0 \quad \text { on } \partial \Omega,
$$

where $|a(x, t)| \leq c_{0}\left(h_{0}(x)+h_{1}(x)|t|^{p-1}\right)$ with $h_{0}(x) \geq 0, h_{1}(x) \geq 1 . a(x, t)$ and $f(x, t)$ are odd with respect to the second variable. Furthermore, $f(x, t)$ is subcritical and satisfies the Ambrosetti-Rabinowitz condition, that is, there exists a constant $\theta>p$ such that

$$
0<\theta \int_{0}^{t} f(x, s) d s \leq f(x, t) t \quad \text { for any } x \in \Omega
$$

The aim of this paper is to obtain infinitely many solutions for the problem (1.1) when $2 p<N$. The main difficulty lies in the fact that the embeddings $W_{0}^{2, p}(\Omega) \hookrightarrow L^{p^{*}}(\Omega)$ and $W_{0}^{2, p}(\Omega) \hookrightarrow L^{p}\left(\Omega,|x|^{-2 p} d x\right)$ are not compact. Furthermore, the assumption $\left(\mathrm{f}_{1}\right)$ is not the usual subcritical growth (1.8). The condition $\left(\mathrm{f}_{3}\right)$ is weaker than the A-R condition (1.10). We use the concentration compactness principle (see $[17,18])$ to overcome those difficulties. The following theorem is our main result.

Theorem 1.1 Assume $f(x, u)$ satisfies $\left(\mathrm{f}_{1}\right)-\left(\mathrm{f}_{4}\right)$, then the problem (1.1) possesses infinitely many weak solutions and the corresponding critical values are positive.

This paper proceeds as follows. In the next section, we prove the energy functional $I(u)$ satisfies the Palais-Smale condition. In Section 3, by using the symmetric mountain pass theorem (see [19]), we get the main result of this paper. Throughout the paper, denote

$$
S_{p, \lambda}:=\inf _{u \in W_{0}^{2, p}(\Omega) \backslash\{0\}} \frac{\|u\|^{p}}{\|u\|_{p^{p}}^{p}} .
$$

We use $\|u\|_{q}=\left(\int_{\Omega}|u|^{q} d x\right)^{1 / q}$ to denote the norm of $L^{q}(\Omega), C, C_{i}$ stand for universal constants. We omit $d x$ and $\Omega$ in the integrals if there is no other indication.

\section{Palais-Smale condition}

To show the (PS) sequence $\left\{u_{n}\right\}$ of the variational functional $I(u)$ is compact in $W_{0}^{2, p}(\Omega)$, we first prove the boundedness of $\left\{u_{n}\right\}$ by the analytic argument which has been used in [20]. Then, using the concentration compactness principle, we get the compactness of $\left\{u_{n}\right\}$.

Lemma 2.1 The condition $\left(\mathrm{f}_{1}\right)$ implies that for any $\varepsilon>0$, there exists a positive constant $C_{\varepsilon}$ such that

$$
F(x, u) \leq \varepsilon|u|^{p^{\prime \prime}}+C_{\varepsilon}|u|, \quad(x, u) \in \Omega \times \mathbb{R} .
$$

$\left(\mathrm{f}_{2}\right)$ implies that $F(x, u)=o\left(u^{p}\right)$ as $u \rightarrow 0$. Furthermore, for any $|u| \geq M>0$, there exists a small positive constant $\theta$

$$
F(x, u) \geq C|u|^{p+\theta} \text { and } f(x, u) u>0 .
$$


Lemma 2.2 Under the conditions $\left(\mathrm{f}_{1}\right),\left(\mathrm{f}_{2}\right)$ and $\left(\mathrm{f}_{3}\right)$, if the sequence $\left\{u_{n}\right\} \in W_{0}^{2, p}(\Omega)$ satisfies

$$
I\left(u_{n}\right) \rightarrow c, \quad I^{\prime}\left(u_{n}\right) \rightarrow 0 \quad \text { as } n \rightarrow+\infty,
$$

then the sequence $\left\{u_{n}\right\}$ is bounded in $W_{0}^{2, p}(\Omega)$.

Proof We prove this lemma by contradiction. Without loss of generality, we assume that

$$
\left\|u_{n}\right\| \rightarrow+\infty \text { as } n \rightarrow+\infty
$$

Set $w_{n}=u_{n} /\left\|u_{n}\right\|$, then $\left\|w_{n}\right\|=1$ for any $n \in \mathbb{N}$. As $n \rightarrow+\infty$, there exists $w \in W_{0}^{2, p}(\Omega)$ such that

$$
\begin{array}{ll}
w_{n} \rightarrow w & \text { weakly in } W_{0}^{2, p}(\Omega), \\
w_{n} \rightarrow w & \text { strongly in } L^{s}(\Omega) \text { for any } 1 \leq s<p^{*}, \\
w_{n} \rightarrow w & \text { a.e. } x \in \Omega .
\end{array}
$$

If $w \not \equiv 0$, we denote $\Omega_{0}=\{x \in \Omega: w=0\}$, set $\Omega \backslash \Omega_{0}$ is nonempty. (2.3) implies that

$$
\left\|u_{n}\right\|^{p}-\int_{\Omega} f\left(x, u_{n}\right) u_{n} d x=o(1)
$$

Therefore,

$$
1-o(1)=\int_{\Omega} \frac{f\left(x, u_{n}\right) u_{n}}{\left\|u_{n}\right\|^{p}} d x=\int_{\Omega_{0}} \frac{f\left(x, u_{n}\right) u_{n}}{\left\|u_{n}\right\|^{p}} d x+\int_{\Omega \backslash \Omega_{0}} \frac{f\left(x, u_{n}\right) u_{n}}{\left\|u_{n}\right\|^{p}} d x
$$

As $n \rightarrow+\infty, \int_{\Omega}\left|u_{n}\right|^{p} d x \leq\left\|u_{n}\right\| \rightarrow 0$. So, $\left|u_{n}\right| \rightarrow+\infty$ for $x \in \Omega \backslash \Omega_{0}$. It follows from $\left(\mathrm{f}_{2}\right)$ that

$$
\lim _{u_{n} \rightarrow \infty} \frac{f\left(x, u_{n}\right) u_{n}}{\left\|u_{n}\right\|^{p}}=\lim _{u_{n} \rightarrow \infty} \frac{f\left(x, u_{n}\right) u_{n}}{\left|u_{n}\right|^{p}}\left|w_{n}\right|^{p}=+\infty .
$$

Since $\left|\Omega \backslash \Omega_{0}\right|>0$, then

$$
\int_{\Omega \backslash \Omega_{0}} \frac{f\left(x, u_{n}\right) u_{n}}{\left\|u_{n}\right\|^{p}} d x \rightarrow+\infty \quad \text { as } n \rightarrow+\infty .
$$

Using (2.1) and (2.2), we derive that

$$
\begin{aligned}
\int_{\Omega_{0}} \frac{f\left(x, u_{n}\right) u_{n}}{\left\|u_{n}\right\|^{p}} d x & =\frac{1}{\left\|u_{n}\right\|^{p}}\left(\int_{\Omega_{0}\left(\left|u_{n}\right|>M\right)} \cdots+\int_{\Omega_{0}\left(\left|u_{n}\right| \leq M\right)} \cdots\right) \\
& \geq-\frac{1}{\left\|u_{n}\right\|^{p}} \int_{\Omega_{0}\left(\left|u_{n}\right| \leq M\right)}\left|f\left(x, u_{n}\right) u_{n}\right| d x \\
& \geq-\frac{C}{\left\|u_{n}\right\|^{p}}\left(M+M^{p^{*}}\right)\left|\Omega_{0}\right| \\
& \geq-C_{1} \quad \text { as } n \rightarrow+\infty .
\end{aligned}
$$


From (2.6) and (2.7), we get

$$
\int_{\Omega} \frac{f\left(x, u_{n}\right) u_{n}}{\left\|u_{n}\right\|^{p}} d x \rightarrow+\infty \quad \text { as } n \rightarrow+\infty
$$

This contradicts (2.5).

If $w \equiv 0$, then $w_{n} \rightarrow 0$ strongly in $L^{1}(\Omega)$. Since $I\left(u_{n}\right) \rightarrow c$, we know that

$$
c \leftarrow \frac{1}{p}\left\|u_{n}\right\|^{p}-\int F\left(x, u_{n}\right) d x \geq \frac{1}{p}\left\|u_{n}\right\|^{p}-\varepsilon \int\left|u_{n}\right|^{p^{*}} d x-C_{\varepsilon} \int\left|u_{n}\right| d x .
$$

In (2.9), choose $\varepsilon=1$, then

$$
\frac{1}{p}\left\|u_{n}\right\|^{p} \leq\left\|u_{n}\right\|_{p^{*}}^{p^{*}}+C_{2}\left\|u_{n}\right\|_{1}+c \leq C_{3}\left\|u_{n}\right\|_{p^{*}}^{p^{*}}+c .
$$

From (2.4), it follows that $\left\|u_{n}\right\|_{p^{*}} \rightarrow+\infty$ as $n \rightarrow+\infty$. Denote

$$
A_{n}=M \frac{\left\|u_{n}\right\|}{\left\|u_{n}\right\|_{p^{*}}} \geq M S_{p, \lambda}^{1 / p}
$$

where $M$ is any fixed positive constant. It is clear that

$$
\frac{A_{n}}{\left\|u_{n}\right\|}=\frac{M}{\left\|u_{n}\right\|_{p^{*}}} \rightarrow 0 \quad(\text { as } n \rightarrow+\infty)
$$

Therefore, for $n$ large enough, $A_{n} /\left\|u_{n}\right\| \in(0,1)$.

For every $n \in \mathbb{N}$, we define a sequence of $t_{n}$ as follows:

$$
I\left(t_{n} u_{n}\right)=\max _{t \in[0,1]} I\left(t u_{n}\right)
$$

We claim that

$$
I\left(t_{n} u_{n}\right) \rightarrow+\infty \quad(\text { as } n \rightarrow+\infty) .
$$

In fact, by (2.1)-(2.10) and the definition of $t_{n}$, we have

$$
\begin{aligned}
I\left(t_{n} u_{n}\right) & \geq I\left(\frac{A_{n}}{\left\|u_{n}\right\|} u_{n}\right) \geq \frac{1}{p} A_{n}^{p}-\varepsilon \int\left|\frac{A_{n}}{\left\|u_{n}\right\|} u_{n}\right|^{p^{*}} d x-C_{\varepsilon} A_{n} \int\left|w_{n}\right| d x \\
& =\frac{1}{p} A_{n}^{p}-\varepsilon M^{p^{\prime \prime}}-C_{\varepsilon} A_{n} \int\left|w_{n}\right| d x .
\end{aligned}
$$

If $A_{n} \rightarrow+\infty$ as $n \rightarrow+\infty$, then (2.11) follows from the above estimate. If $A_{n}$ is bounded for any $n$, that is, there exists a constant $K>0$ such that $A_{n} \leq K$, then

$$
\begin{aligned}
I\left(t_{n} u_{n}\right) & \geq \frac{1}{p} A_{n}^{p}-\varepsilon M^{p^{*}}-C_{\varepsilon} A_{n} \int\left|w_{n}\right| d x \geq \frac{1}{p} S_{p, \lambda} M^{p}-\varepsilon M^{p^{*}}-C_{\varepsilon} K \int\left|w_{n}\right| d x \\
& \rightarrow \frac{1}{p} S_{p, \lambda} M^{p}-\varepsilon M^{p^{*}} \quad \text { as } n \rightarrow+\infty,
\end{aligned}
$$


where we use the fact that $\left\|w_{n}\right\|_{1} \rightarrow 0$ as $n \rightarrow+\infty$. The positive constant $\varepsilon$ is arbitrary, thus

$$
\lim _{n \rightarrow+\infty} I\left(t_{n} u_{n}\right) \geq \frac{1}{p} S_{p, \lambda} M^{p} \quad \text { for any positive constant } M .
$$

(2.11) follows from the above inequality.

From (2.3), we know that

$$
p c+o(1)=p I\left(u_{n}\right)-\left\langle I^{\prime}\left(u_{n}\right), u_{n}\right\rangle=\int\left(f\left(x, u_{n}\right) u_{n}-p F\left(x, u_{n}\right)\right) d x
$$

By the definition of $t_{n}$, we obtain $\left.\frac{d}{d t} I\left(t u_{n}\right)\right|_{t=t_{n}}=0$ and $t_{n} \in[0,1]$, then $\left|t_{n} u_{n}\right| \leq\left|u_{n}\right|$. The condition $\left(f_{3}\right)$ and (2.11) derive that

$$
\begin{aligned}
\int\left(f\left(x, u_{n}\right) u_{n}-p F\left(x, u_{n}\right)\right) d x & \geq \int\left(f\left(x, t_{n} u_{n}\right) t_{n} u_{n}-p F\left(x, t_{n} u_{n}\right)\right) d x \\
& =p I\left(t_{n} u_{n}\right)-\left\langle I^{\prime}\left(t_{n} u_{n}\right), t_{n} u_{n}\right\rangle \\
& =p I\left(t_{n} u_{n}\right)-\left.t_{n} \frac{d}{d t} I\left(t u_{n}\right)\right|_{t=t_{n}} \\
& \rightarrow+\infty
\end{aligned}
$$

Which is a contradiction to (2.12). Therefore, the sequence $\left\{u_{n}\right\}$ is bounded in $W_{0}^{2, p}(\Omega)$.

Under the conditions of Lemma 2.2, we know $\left\|u_{n}\right\| \leq C$. Therefore, there exists a subsequence, still denoted by $\left\{u_{n}\right\}$, and some $u \in W_{0}^{2, p}(\Omega)$ such that

$$
\begin{array}{ll}
u_{n} \rightarrow u & \text { weakly in } W_{0}^{2, p}(\Omega), \\
u_{n} \rightarrow u & \text { weakly in } L^{p}\left(\Omega,|x|^{-2 p} d x\right), \\
u_{n} \rightarrow u & \text { strongly in } L^{s}(\Omega) \text { for any } 1 \leq s<p^{*}, \\
u_{n} \rightarrow u & \text { a.e. } x \in \Omega .
\end{array}
$$

Obviously, $u$ is a weak solution of (1.1). Now we prove that $u \neq \equiv 0$. According to the concentration compactness principle (see $[17,18]$ ), there exists a subsequence, still denoted by $\left\{u_{n}\right\}$, at most countable set $J$, a set of different points $\left\{x_{j}\right\}_{j \in J} \subset \bar{\Omega} \backslash\{0\}$ and two positive number sequences $\left\{\mu_{j}\right\}_{j \in J \cup\{0\}},\left\{v_{j}\right\}_{j \in J \cup\{0\}}$ such that

$$
\begin{aligned}
& \left|\Delta u_{n}\right|^{p} \rightarrow d \mu \geq|\Delta u|^{p}+\sum_{j \in J} \mu_{j} \delta_{x_{j}}+\mu_{0} \delta_{0} \quad \text { weakly in the sense of measure, } \\
& \left|u_{n}\right|^{p^{*}} \rightarrow d v=|u|^{p^{*}}+\sum_{j \in J} v_{j} \delta_{x_{j}}+v_{0} \delta_{0} \quad \text { weakly in the sense of measure, } \\
& \frac{\left|u_{n}\right|^{p}}{|x|^{2 p}} \rightarrow d \gamma=\frac{|u|^{p}}{|x|^{2 p}}+\gamma_{0} \delta_{0} \quad \text { weakly in the sense of measure, } \\
& S_{p, \lambda} v_{0}^{\frac{p}{p^{*}}} \leq \mu_{0}-\lambda \gamma_{0}, \quad \text { and } \quad S_{p, 0} v_{j}^{\frac{p}{p^{*}}} \leq \mu_{j} \quad \text { for } j \in J
\end{aligned}
$$

where $\delta_{x_{j}}$ is the unit Dirac measure at $x_{j}$. 
Lemma 2.3 Assume $f(x, u)$ satisfies $\left(f_{1}\right)$, the sequence $\left\{u_{n}\right\}$ is bounded in $W_{0}^{2, p}(\Omega)$ and satisfies (2.3). Then the set $J=\left\{x_{1}, x_{2}, \ldots, x_{j}\right\} \subset \bar{\Omega}$ is finite, $\mu_{0}-\lambda \gamma_{0}=0$.

Proof We first prove that $\mu_{j}=0$ for any $x_{j} \in J$.

Let $\varepsilon>0$ be small enough such that $0 \notin B_{\varepsilon}\left(x_{j}\right)$ and $B_{\varepsilon}\left(x_{i}\right) \cap B_{\varepsilon}\left(x_{j}\right)=\emptyset$ for $i \neq j$. $\phi_{j}(x) \in[0,1]$ is a cutting-off function in $\mathcal{C}_{0}^{\infty}(\Omega)$. $\phi_{j}(x) \equiv 1$ for $\left|x-x_{j}\right|<\varepsilon / 2, \phi_{j}(x) \equiv 0$ for $\left|x-x_{j}\right| \geq \varepsilon$, and $\left|\Delta \phi_{j}\right| \leq 4 / \varepsilon^{2}$. Since

$$
\begin{aligned}
\left\langle I^{\prime}\left(u_{n}\right), u_{n} \phi_{j}\right\rangle= & \int\left|\Delta u_{n}\right|^{p} \phi_{j} d x+2 \int\left|\Delta u_{n}\right|^{p-2} \Delta u_{n} \nabla u_{n} \nabla \phi_{j} d x \\
& +\int u_{n}\left|\Delta u_{n}\right|^{p-2} \Delta u_{n} \Delta \phi_{j} d x \\
& -\int \lambda \frac{\left|u_{n}\right|^{p}}{|x|^{2 p}} \phi_{j} d x-\int f\left(x, u_{n}\right) u_{n} \phi_{j} d x,
\end{aligned}
$$

we deduce that

$$
\begin{aligned}
& \lim _{\varepsilon \rightarrow 0} \lim _{n \rightarrow+\infty} \int\left|\Delta u_{n}\right|^{p} \phi_{j} d x=\lim _{\varepsilon \rightarrow 0} \int \phi_{j} d \mu \geq \lim _{\varepsilon \rightarrow 0} \int|\Delta u|^{p} \phi_{j} d x+\mu_{j}=\mu_{j}, \\
& \lim _{\varepsilon \rightarrow 0} \lim _{n \rightarrow+\infty}\left|\int \frac{\left|u_{n}\right|^{p}}{|x|^{2 p}} \phi_{j} d x\right| \leq \lim _{\varepsilon \rightarrow 0} \lim _{n \rightarrow+\infty} \int_{B_{\varepsilon}\left(x_{j}\right)} \frac{\left|u_{n}\right|^{p}}{\left(\left|x_{j}\right|-\varepsilon\right)^{2 p}}\left|\phi_{j}\right| d x=0, \\
& \lim _{\varepsilon \rightarrow 0} \lim _{n \rightarrow+\infty} \int\left|u_{n}\right|^{p^{*}} \phi_{j} d x=\lim _{\varepsilon \rightarrow 0} \int|u|^{p^{*}} \phi_{j} d x+v_{j}=v_{j}, \\
& \lim _{\varepsilon \rightarrow 0} \lim _{n \rightarrow+\infty} \int\left|u_{n}\right| \phi_{j} d x=\lim _{\varepsilon \rightarrow 0} \int|u| \phi_{j} d x=0 .
\end{aligned}
$$

By Hölder’s inequality,

$$
\begin{aligned}
& \left.\lim _{\varepsilon \rightarrow 0} \lim _{n \rightarrow+\infty}\left|\int\right| \Delta u_{n}\right|^{p-2} \Delta u_{n} \nabla u_{n} \nabla \phi_{j} d x \mid \\
& \quad \leq \lim _{\varepsilon \rightarrow 0} \lim _{n \rightarrow+\infty}\left\|\Delta u_{n}\right\|_{L^{p}\left(B_{\varepsilon}\left(x_{j}\right)\right)}^{p-1}\left\|\nabla u_{n}\right\|_{L^{N p /(N-p)\left(B_{\varepsilon}\left(x_{j}\right)\right)}}\left\|\nabla \phi_{j}\right\|_{L^{N}\left(B_{\varepsilon}\left(x_{j}\right)\right)} \\
& \quad \leq C \lim _{\varepsilon \rightarrow 0}\left\|\Delta u_{n}\right\|_{L^{p}\left(B_{\varepsilon}\left(x_{j}\right)\right)}^{p}=0 \\
& \left.\lim _{\varepsilon \rightarrow 0} \lim _{n \rightarrow+\infty}\left|\int\right| \Delta u_{n}\right|^{p-2} \Delta u_{n} u_{n} \Delta \phi_{j} d x \mid \\
& \quad \leq \lim _{\varepsilon \rightarrow 0} \lim _{n \rightarrow+\infty}\left\|\Delta u_{n}\right\|_{L^{p}\left(B_{\varepsilon}\left(x_{j}\right)\right)}^{p-1}\left\|u_{n}\right\|_{L^{p^{n}}\left(B_{\varepsilon}\left(x_{j}\right)\right)}\left\|\Delta \phi_{j}\right\|_{L^{N / 2}\left(B_{\varepsilon}\left(x_{j}\right)\right)} \\
& \quad \leq C \lim _{\varepsilon \rightarrow 0}\left\|u_{n}\right\|_{L^{p^{*}}\left(B_{\varepsilon}\left(x_{j}\right)\right)}=0 .
\end{aligned}
$$

Using $\left(f_{1}\right),(2.14)$ and $(2.15)$, we have

$$
\lim _{\varepsilon \rightarrow 0} \lim _{n \rightarrow+\infty} \int\left|f\left(x, u_{n}\right) u_{n} \phi_{j}\right| d x \leq \lim _{\varepsilon \rightarrow 0} \lim _{n \rightarrow+\infty}\left(\varepsilon \int\left|u_{n}\right|^{p^{\prime}} \phi_{j} d x+C_{\varepsilon} \int\left|u_{n}\right| \phi_{j} d x\right)=0 .
$$

By the above estimates, (2.13) becomes

$$
0=\lim _{\varepsilon \rightarrow 0} \lim _{n \rightarrow+\infty}\left\langle I^{\prime}\left(u_{n}\right), u_{n} \phi_{j}\right\rangle \geq \mu_{j} .
$$

Therefore, for any $j \in J, \mu_{j}=0$, which implies that $J$ is finite. 
If the concentration is at the origin, let $\phi_{0}(x) \in[0,1]$ be a cutting-off function in $\mathcal{C}_{0}^{\infty}(\Omega)$. $\phi_{0}(x) \equiv 1$ for $|x|<\varepsilon / 2, \phi_{0}(x) \equiv 0$ for $|x| \geq \varepsilon$, and $\left|\Delta \phi_{0}\right| \leq 4 / \varepsilon^{2}$. Choose $\varepsilon>0$ small enough such that $x_{j} \notin B_{\varepsilon}(0)$ for all $j \in J$. Then

$$
\begin{aligned}
& \lim _{\varepsilon \rightarrow 0} \lim _{n \rightarrow+\infty} \int\left|\Delta u_{n}\right|^{p} \phi_{0} d x=\lim _{\varepsilon \rightarrow 0} \int \phi_{0} d \mu \geq \lim _{\varepsilon \rightarrow 0} \int\left|\Delta u_{n}\right|^{p} \phi_{0} d x+\mu_{0}=\mu_{0}, \\
& \lim _{\varepsilon \rightarrow 0} \lim _{n \rightarrow+\infty}\left|\int \frac{\left|u_{n}\right|^{p}}{|x|^{2 p}} \phi_{0} d x\right|=\lim _{\varepsilon \rightarrow 0} \int_{B_{\varepsilon}\left(x_{j}\right)} \frac{|u|^{p}}{|x|^{2 p}}\left|\phi_{0}\right| d x+\gamma_{0}=\gamma_{0} .
\end{aligned}
$$

Similarly, we can get

$$
\begin{aligned}
& \lim _{\varepsilon \rightarrow 0} \lim _{n \rightarrow+\infty} \int\left(2\left|\Delta u_{n}\right|^{p-2} \Delta u_{n} \nabla u_{n} \nabla \phi_{0}+\left|\Delta u_{n}\right|^{p-2} \Delta u_{n} u_{n} \Delta \phi_{j}\right) d x=0, \\
& \lim _{\varepsilon \rightarrow 0} \lim _{n \rightarrow+\infty} \int\left|f\left(x, u_{n}\right) u_{n} \phi_{0}\right| d x=0 .
\end{aligned}
$$

From equalities (2.17)-(2.20), we get

$$
0=\lim _{\varepsilon \rightarrow 0} \lim _{n \rightarrow+\infty}\left\langle I^{\prime}\left(u_{n}\right), u_{n} \phi_{0}\right\rangle \geq \mu_{0}-\lambda \gamma_{0} .
$$

On the other hand, $\mu_{0}-\lambda \gamma_{0} \geq S_{p, \lambda} v_{0}^{\frac{p}{p^{p}}} \geq 0$, thus $\mu_{0}-\lambda \gamma_{0}=0$. The proof is complete.

Lemma 2.4 Assume $f(x, u)$ satisfies $\left(\mathrm{f}_{1}\right)$, the sequence $\left\{u_{n}\right\}$ satisfying $(2.3)$ is bounded in $W_{0}^{2, p}(\Omega)$. Then there exist some $u \in W_{0}^{2, p}(\Omega)$ and a subsequence, still denoted by $\left\{u_{n}\right\}$, such that

$$
\begin{aligned}
& u_{n} \rightarrow u \quad \text { strongly in } L^{p^{*}}\left(\Omega_{\epsilon}\right), \\
& \nabla u_{n} \rightarrow \nabla u \quad \text { strongly in } L^{\frac{N p}{N-p}}\left(\Omega_{\epsilon}\right),
\end{aligned}
$$

where $\Omega_{\epsilon}=\Omega \backslash \bigcup_{i=0}^{j} \bar{B}_{\epsilon}\left(x_{i}\right), x_{i} \in J=\left\{x_{1}, x_{2}, \ldots, x_{j}\right\} \cup\{0\}$.

Proof Lemma 2.3 implies that $J$ is finite. Choose $\varphi \in C_{0}^{\infty}(\Omega)$ with $\varphi\left(x_{i}\right)=0$ for any $x_{i} \in$ $J \cup\{0\}$. Then

$$
\begin{aligned}
\int\left|\varphi u_{n}\right|^{p^{\circ}} d x & =\int|\varphi u|^{p^{\circ}} d x+\sum_{i=1}^{j} v_{i} \varphi^{p^{\circ}}\left(x_{i}\right)+\nu_{0} \varphi^{p^{\circ}}(0) \\
& =\int|\varphi u|^{p^{\circ}} d x .
\end{aligned}
$$

Since $\varphi u_{n} \rightarrow \varphi u$ a.e. in $\Omega$, we get that

$$
u_{n} \rightarrow u \quad \text { strongly in } L^{p^{*}}\left(\Omega_{\epsilon}\right) .
$$

Similarly,

$$
\nabla u_{n} \rightarrow \nabla u \quad \text { strongly in } L^{\frac{N p}{N-p}}\left(\Omega_{\epsilon}\right) .
$$


By using the Lebesgue decomposition theorem, we have

$$
d \mu-\lambda d \gamma=|\Delta u|^{p}-\lambda \frac{|u|^{p}}{|x|^{2 p}}+d \sigma
$$

where $\left(|\Delta u|^{p}-\lambda|u|^{p}|x|^{-2 p}\right) \perp d \sigma$. Then $d \sigma \geq \sum_{j \in J} \mu_{j} \delta_{x_{j}}+\left(\mu_{0}-\lambda \gamma_{0}\right) \delta_{0}$.

Lemma 2.5 Assume $f(x, u)$ satisfies $\left(\mathrm{f}_{1}\right)-\left(\mathrm{f}_{3}\right)$, the sequence $\left\{u_{n}\right\} \in W_{0}^{2, p}(\Omega)$ satisfies (2.3). Then there exist $u \in W_{0}^{2, p}(\Omega)$ and a subsequence, still denoted by $\left\{u_{n}\right\}$, such that $u_{n}$ converges to $u$ strongly in $W_{0}^{2, p}(\Omega)$.

Proof We first prove that

$$
\left(\sigma-\sum_{j \in J} \mu_{j} \delta_{x_{j}}-\left(\mu_{0}-\lambda \gamma_{0}\right) \delta_{0}\right)(\bar{\Omega})=0
$$

Claim for any closed set $F \subset \bar{\Omega} \backslash \bar{J}, \bar{J}=J \cup\{0\}, \sigma(F)=0$. In fact, denote $r=\operatorname{dist}(F, \bar{J})>0$. Then, by using a finite covering theorem, there exist finite open balls $B_{r / 4}\left(z_{i}\right)$, with $z_{i} \in F$, $i=1, \ldots, m$, such that $F \subset \bigcup_{i=1}^{m} B_{r / 4}\left(z_{i}\right) \subset \bar{\Omega} \backslash \bar{J}$. Let $\varrho(t)$ be a smooth cutting-off function. $0 \leq \varrho(t) \leq 1$ for any $t \in[0, \infty) . \varrho(x) \equiv 1$ for $0 \leq t \leq 1 / 2$, and $\varrho \equiv 0$ for $t \geq 1$. Denote $\eta_{\varepsilon, y}=$ $\varrho(|x-y| / \varepsilon)$, then

$$
\begin{aligned}
& \int_{\mathbb{R}^{N}}\left|\nabla \eta_{\varepsilon, y}\right|^{N} d x=\int_{1 / 2}^{1}\left|\varrho^{\prime}(t)\right|^{N} d t=C_{1}, \\
& \int_{\mathbb{R}^{N}}\left|\Delta \eta_{\varepsilon, y}\right|^{\frac{N}{2}} d x:=\int_{1 / 2}^{1}\left|\varrho^{\prime \prime}(t)\right|^{\frac{N}{2}} d t=C_{2} .
\end{aligned}
$$

The sequence $\left\{\eta_{\varepsilon, y} u_{n}\right\}$ is still bounded in $W_{0}^{2, p}(\Omega)$. Define

$$
\eta(x)=\sum_{i=1}^{m} \eta_{r / 4, z_{i}}(x)
$$

then $\eta(x) \in C_{0}^{\infty}(\bar{\Omega} \backslash \bar{J})$ and $0 \leq \eta(x) \leq m$. Furthermore,

$$
F \subset \bigcup_{i=1}^{m} B_{\frac{r}{4}}\left(z_{i}\right) \subset \operatorname{spt} \eta(x) \subset \Omega_{\frac{r}{4}},
$$

where spt $\eta(x)$ denotes the support of $\eta(x)$. As $n \rightarrow+\infty,(2.3)$ implies

$$
\begin{aligned}
0 \leftarrow & \left\langle I^{\prime}\left(u_{n}\right)-I^{\prime}(u),\left(u_{n}-u\right) \eta\right\rangle \\
= & \int_{\Omega} \eta\left(\left|\Delta u_{n}\right|^{p}-\left|\Delta u_{n}\right|^{p-2} \Delta u_{n} \Delta u-|\Delta u|^{p-2} \Delta u \Delta u_{n}+|\Delta u|^{p}\right) d x \\
& -\lambda \int_{\Omega} \frac{\eta}{|x|^{2 p}}\left(\left|u_{n}\right|^{p}-\left|u_{n}\right|^{p-2} u_{n} u-|u|^{p-2} u u_{n}+|u|^{p}\right) d x \\
& +2 \int_{\Omega} \nabla \eta \nabla\left(u_{n}-u\right)\left(\left|\Delta u_{n}\right|^{p-2} \Delta u_{n}-|\Delta u|^{p-2} \Delta u\right) d x \\
& +\int_{\Omega} \Delta \eta\left(u_{n}-u\right)\left(\left|\Delta u_{n}\right|^{p-2} \Delta u_{n}-|\Delta u|^{p-2} \Delta u\right) d x
\end{aligned}
$$


Xis and Wang Journal of Inequalities and Applications 2013, 2013:9

Page 10 of 13

http://www.journalofinequalitiesandapplications.com/content/2013/1/9

$$
\begin{aligned}
& -\int_{\Omega} \eta\left(u_{n}-u\right)\left(f\left(x, u_{n}\right)-f(x, u)\right) d x \\
= & I+I I+I I I+I V+V .
\end{aligned}
$$

As $n \rightarrow+\infty$,

$$
\begin{aligned}
I+I I= & \int_{\Omega} \eta\left(\left|\Delta u_{n}\right|^{p}-\left|\Delta u_{n}\right|^{p-2} \Delta u_{n} \Delta u-|\Delta u|^{p-2} \Delta u \Delta u_{n}+|\Delta u|^{p}\right) d x \\
& -\lambda \int_{\Omega} \frac{\eta}{|x|^{2 p}}\left(\left|u_{n}\right|^{p}-\left|u_{n}\right|^{p-2} u_{n} u-|u|^{p-2} u u_{n}+|u|^{p}\right) d x \\
= & \int_{\Omega} \eta\left(\left|\Delta u_{n}\right|^{p}-|\Delta u|^{p}-\lambda \frac{\left|u_{n}\right|^{p}-|u|^{p}}{|x|^{2 p}}\right) d x+o(1) \\
= & \int_{\Omega} \eta d \sigma+o(1),
\end{aligned}
$$

where we have used (2.23). Lemma 2.2 implies that $\left\|u_{n}\right\| \leq M$. Hölder's inequality, (2.25)(2.28) and Lemma 2.4 deduce that

$$
\begin{aligned}
|I I I| & =2\left|\int_{\Omega} \nabla \eta \nabla\left(u_{n}-u\right)\left(\left|\Delta u_{n}\right|^{p-2} \Delta u_{n}-|\Delta u|^{p-2} \Delta u\right) d x\right| \\
& \leq C \int_{\Omega}\left(\left|\Delta u_{n}\right|^{p}+|\Delta u|^{p}\right) d x\|\nabla \eta\|_{N}\left(\int_{\mathrm{spt}(\eta)}\left|\nabla\left(u_{n}-u\right)\right|^{\frac{N p}{N-p}} d x\right)^{\frac{N-p}{N p}} \\
& \leq C(m, M, p) C_{1}\left(\int_{\Omega_{\frac{r}{4}}}\left|\nabla\left(u_{n}-u\right)\right|^{\frac{N p}{N-p}} d x\right)^{\frac{N-p}{N p}} \\
& \rightarrow 0 \text { as } n \rightarrow+\infty, \\
|I V| & =\left|\int_{\Omega}\left(u_{n}-u\right) \Delta \eta\left(\left|\Delta u_{n}\right|^{p-2} \Delta u_{n}-|\Delta u|^{p-2} \Delta u\right) d x\right| \\
& \leq C(m, M, p) C_{2}\left(\int_{\Omega \frac{r}{4}}\left|u_{n}-u\right|^{p^{*}} d x\right)^{\frac{1}{p^{*}}} \\
& \rightarrow 0 \text { as } n \rightarrow+\infty .
\end{aligned}
$$

By (2.1), for any $\varepsilon>0$, we have

$$
\begin{aligned}
|V| & =\left|\int_{\Omega}\left(f\left(x, u_{n}\right)-f(x, u)\right)\left(u_{n}-u\right) \eta d x\right| \\
& \leq \int_{\Omega}\left(\left|f\left(x, u_{n}\right)\right|+|f(x, u)|\right)\left|u_{n}-u\right| \eta d x \\
& \leq \int_{\Omega}\left(C_{\varepsilon}+\varepsilon\left(\left|u_{n}\right|^{p^{*}-1}+|u|^{p^{*}-1}\right)\right)\left|u_{n}-u\right| \eta d x \\
& \leq C_{\varepsilon} \int_{\Omega}\left|u_{n}-u\right| \eta d x+\varepsilon\left(\left\|u_{n}\right\|_{p^{p^{*}}}^{p^{*}-1}+\|u\|_{p^{*}}^{p^{*}-1}\right)\left(\int_{\mathrm{spt}(\eta)}\left|u_{n}-u\right|^{p^{*}} d x\right)^{\frac{1}{p^{*}}} \\
& \leq C_{\varepsilon} \int_{\Omega}\left|u_{n}-u\right| d x+\varepsilon C(M) \\
& \rightarrow 0 \quad \text { as } n \rightarrow+\infty, \varepsilon \rightarrow 0 .
\end{aligned}
$$


Therefore, as $n \rightarrow+\infty$,

$$
0 \leq \sigma(F) \leq \sigma(\operatorname{spt}(\eta)) \leq \int_{\Omega} \eta d \sigma \leq|I I I|+|I V|+|V|=0
$$

Since the set $F$ is arbitrary, (2.24) is obtained.

Lemma 2.3 means that $\mu_{j}=0$ for any $j \in J$ and $\mu_{0}-\lambda \gamma_{0}=0$, so $\sigma(\bar{\Omega})=0$, which implies that as $n \rightarrow+\infty$,

$$
\int_{\Omega}\left(\left|\Delta u_{n}\right|^{p}-\lambda \frac{\left|u_{n}\right|^{p}}{|x|^{2 p}}\right) d x \rightarrow \int_{\Omega}\left(|\Delta u|^{p}-\lambda \frac{|u|^{p}}{|x|^{2 p}}\right) d x .
$$

Together with $u_{n} \rightarrow u$ a.e. in $\Omega$, we complete the proof.

\section{The proof of the main result}

In this section, by using the following symmetric mountain pass theorem (see [19]), we give the proof of Theorem 1.1.

Lemma 3.1 (Symmetric mountain pass theorem) Assume functional I satisfies the following conditions:

(1) $I \in \mathcal{C}^{1}\left(W_{0}^{2, p}(\Omega), \mathbb{R}\right)$ is even and satisfies the Palais-Smale condition.

(2) There exists a finite dimensional subspace $X \in W_{0}^{2, p}(\Omega)$ such that $\left.I\right|_{X \cap \partial B_{r}} \geq a>0$.

(3) There exists a sequence of the finite dimensional subspace $\left\{X_{j}\right\}, \operatorname{dim}\left(X_{j}\right)=j$ and $r_{j}>0$ such that

$$
I(u) \leq 0 \quad \text { for any } u \in X_{j} \backslash B_{r_{j}}, j=1,2, \ldots
$$

Then I has infinitely many different critical points, and the corresponding energy values are positive.

Proof of Theorem 1.1 We know the energy functional $I(u)$ is continuous and even. Lemma 2.5 implies that $I(u)$ satisfies the PS condition in $W_{0}^{2, p}(\Omega)$. In any finite dimensional subspace $X \subset W_{0}^{2, p}(\Omega)$, all norms are equivalent. For any $u \in X$, since $F(x, u)=o\left(u^{p}\right)$ as $u \rightarrow 0$, there exists a constant $q>p$ such that

$$
\begin{aligned}
I(u) & =\frac{1}{p} \int_{\Omega}\left(|\Delta u|^{p}-\lambda \frac{|u|^{p}}{|x|^{2 p}}\right) d x-\int_{\Omega} F(x, u) d x \\
& \geq \frac{1}{p}\|u\|^{p}-C\|u\|^{q}>0 \quad \text { for } u \text { small enough. }
\end{aligned}
$$

Thus, there exists $r>0$ such that $\left.I\right|_{X \cap \partial B_{r}} \geq a>0$. On the other hand, by using (2.2), we have

$$
\begin{aligned}
I(u) & =\frac{1}{p} \int_{\Omega}\left(|\Delta u|^{p}-\lambda \frac{|u|^{p}}{|x|^{2 p}}\right) d x-\int_{\Omega} F(x, u) d x \\
& \leq \frac{1}{p}\|u\|^{p}-C \int_{\{x \in \Omega:|u|>M\}}|u|^{p+\theta} d x-C^{\prime} \\
& \leq \frac{1}{p}\|u\|^{p}-C\|u\|^{p+\theta}-C^{\prime} \rightarrow-\infty \quad \text { as } u \rightarrow \infty .
\end{aligned}
$$


There exists a sequence of the finite dimensional subspace $\left\{X_{j}\right\}, \operatorname{dim}\left(X_{j}\right)=j$ and $r_{j}>0$ such that

$$
I(u) \leq 0 \quad \text { for any } u \in X_{j} \backslash B_{r_{j}}, j=1,2, \ldots
$$

Finally, all the assumptions of Lemma 3.1 are satisfied. Hence, the problem (1.1) possesses infinitely many weak solutions, and the corresponding critical values are positive.

Remark 3.1 Under the same conditions of Theorem 1.1, we can also prove that the following $p$-harmonic type equation with Navier boundary conditions:

$$
\begin{cases}\Delta\left(|\Delta u|^{p-2} \Delta u\right)-\lambda \frac{|u|^{p-2} u}{|x|^{2 p}}=f(x, u), & x \in \Omega, \\ u=\Delta u=0, & x \in \partial \Omega\end{cases}
$$

has infinitely many solutions $\left\{u_{n}\right\} \in W^{2, p}(\Omega) \cap W_{0}^{1, p}(\Omega)$, where $\Omega$ containing the origin is an open bounded domain in $\mathbb{R}^{N}, \partial \Omega$ is smooth. $1<p<\frac{N}{2}, 0 \leq \lambda<\bar{\lambda}=[N(p-1)(N-$ $\left.2 p) / p^{2}\right]^{p}$.

Remark 3.2 All the results obtained above obviously hold if we choose $f(x, u)=|u|^{q-2} u+$ $|u|^{r-2} u$ with $p<q<r<p^{*}$.

\section{Competing interests}

The authors declare that they have no competing interests.

\section{Authors' contributions}

HX carried out the existence of infinitely many solutions for the $p$-harmonic equation studies and drafted the manuscript JW participated in its coordination. All authors read and approved the final manuscript.

\section{Author details}

'Department of Mathematics, Henan University of Economics and Law, Zhengzhou, 450002, China. ${ }^{2}$ College of Information and Management Science, Henan Agricultural University, Zhengzhou, 450002, China.

\section{Acknowledgements}

The authors are thankful to the referees for their helpful suggestions and necessary corrections in the completion of this paper. This research is supported by the Education Department of Henan Province (12B110002) and NSF of China (61143002).

Received: 19 May 2012 Accepted: 13 November 2012 Published: 6 January 2013

\section{References}

1. Davies, E, Hinz, A: Explicit constants for Rellich inequalities in $L^{p}(\Omega)$. Math. Z. 227, 511-523 (1998)

2. Mitidieri, E: A simple approach to Hardy's inequalities. Math. Notes 67, 479-486 (2000)

3. Lazer, A, McKenna, P: Large amplitude periodic oscillations in suspension bridges: some new connections with nonlinear analysis. SIAM Rev. 32, 537-578 (1990)

4. Bernis, F, Azorero, JG, Peral, I: Existence and multiplicity of nontrivial solutions in semilinear critical problems of fourth order. Adv. Differ. Equ. 1, 219-240 (1996)

5. Bonder, J, Rossi, J: A fourth order elliptic equation with nonlinear boundary conditions. Nonlinear Anal. 49, 1037-1047 (2002)

6. Deng, Y, Wang, G: On inhomogeneous biharmonic equations involving critical exponents. Proc. R. Soc. Edinb. 129, 925-946 (1999)

7. Deng, Y, Yang, J: Existence of multiple solutions and bifurcations for critical semilinear biharmonic equation. Syst. Sci. Math. Sci. 8, 319-326 (1995)

8. Liu, X, Li, W: Existence and multiplicity of solutions for fourth-order boundary value problems with parameters. J. Math. Anal. Appl. 327, 362-375 (2007)

9. Zeng, $X$, Deng, Y: Existence of multiple solutions for a semilinear biharmonic equation with critical exponent. Acta Math. Sci. 20(4), 547-554 (2000)

10. Zhang, J, Li, S: Multiple nontrivial solutions for some fourth-order semilinear elliptic problems. Nonlinear Anal. 60 , $221-230(2005)$ 
11. Liu, S, Squassina, M: On the existence of solutions to a fourth-order quasilinear resonant problem. Abstr. Appl. Anal. 7(3), 125-133 (2002)

12. Li, C, Tang, C: Three solutions for a Navier boundary value problem involving the $p$-biharmonic. Nonlinear Anal. 72 , 1339-1347 (2010)

13. Ricceri, B: A three critical points theorem revisited. Nonlinear Anal. 70, 3084-3089 (2009)

14. Candito, P, Bisci, G: Multiple solutions for a Navier boundary value problem involving the $p$-biharmonic operator. Discrete Contin. Dyn. Syst. 5, 741-751 (2012)

15. Yang, Z, Geng, D, Yan, H: Existence of nontrivial solutions in $p$-biharmonic problems with critical growth. Chin. Ann. Math., Ser. A 27(1), 129-142 (2006)

16. Wang, W, Zhao, P: Nonuniformly nonlinear elliptic equations of $p$-biharmonic type. J. Math. Anal. Appl. 348, 730-738 (2008)

17. Lions, PL: The concentration-compactness principle in the calculus of variations: the limit case, part 1. Rev. Mat. Iberoam. 1(1), 145-201 (1985)

18. Lions, PL: The concentration-compactness principle in the calculus of variations: the limit case, part 2. Rev. Mat. Iberoam. 1(2), 45-121 (1985)

19. Chang, K: Critical Point Theory and Its Applications. Shanghai Sci. Technol., Shanghai (1986) (in Chinese)

20. Geng, D: Infinitely many solutions of $p$-Laplacian equations with limit subcritical growth. Appl. Math. Mech. 28(10), 1373-1382 (2007)

doi:10.1186/1029-242X-2013-9

Cite this article as: Xie and Wang: Infinitely many solutions for $p$-harmonic equation with singular term. Journal of Inequalities and Applications 2013 2013:9.

\section{Submit your manuscript to a SpringerOpen ${ }^{\circ}$ journal and benefit from:}

- Convenient online submission

Rigorous peer review

- Immediate publication on acceptance

- Open access: articles freely available online

- High visibility within the field

- Retaining the copyright to your article 